\title{
Intravesical Loss of OnabotulinumtoxinA During Endoscopic Intradetrusor Injection - A Multicenter Experience
}

\author{
Vladimír Šámal ${ }^{1,2}$, Jan Krhut ${ }^{3,4}$, Vít Paldus ${ }^{1}$, Jana Müllerová ${ }^{5}, J^{\prime}$ Mečl $^{1}$ \\ ${ }^{1}$ Department of Urology, Regional Hospital Liberec, Liberec, Czech Republic \\ ${ }^{2}$ Department Of Urology, Medical Faculty Charles University, Hradec Králové, Czech Republic \\ ${ }^{3}$ Department Of Urology, University Hospital Ostrava, Ostrava, Czech Republic \\ ${ }^{4}$ Department of Surgical Studies, Ostrava University, Ostrava, Czech Republic \\ ${ }^{5}$ Department of Chemistry, Faculty of Science, Humanities and Education, Technical University of Liberec, Liberec, Czech Republic
}

Purpose: To evaluate intravesical loss of onabolunumtoxinA (onaBTA) during endoscopic injection into the bladder wall for treatment of neurogenic detrusor overactivity (NDO). The intraluminal loss of onaBTA cannot be determined directly, therefore we added methylene blue (MB) to reconstitute onaBTA. Subsequently we used spectrophotometry to determine the total amount of $\mathrm{MB}$ in the irrigation fluid, which allowed us to calculate total intraluminal loss of onaBTA.

Methods: The study population was consisted of 48 patients with NDO. The mean age was $36.6 \pm 8.9$ years. Forty-two patients suffered from NDO due to spinal cord injury and 6 patients suffered from multiple sclerosis. Each patient received 200 units of onaBTA administered by 30 endoscopic injections ( $1 \mathrm{~mL}$ per injection) using a $23-\mathrm{G}$ needle. The entire volume of irrigation fluid was collected and spectrophotometry was used to determine the MB concentration. The total amount of injected onaBTA, total amount of irrigation solution and the known amount of $\mathrm{MB}$ used during reconstitution, allowed for the determination of intravesical loss of onaBTA.

Results: Forty-five patients were included in the final analysis. The mean volume of irrigation fluid was $603.33 \pm 400.14 \mathrm{~mL}$. The mean absorbance was $0.14 \pm 0.12$ with the mean MB concentration $0.19 \pm 0.18 \mathrm{mg} / \mathrm{L}$. The mean calculated loss of onaBTA was $4.14 \pm 4.11$ units.

Conclusions: The endoscopic injection of onaBTA marked by MB into the bladder wall is associated with minimal intravesical loss of the agent, representing less than $3 \%$ of the administered dose of MB. This may reflect the amount of the onaBTA detected in the irrigation fluid.

Keywords: Botulinum toxins, Type A; Urinary bladder, Neurogenic; Intravesical injection; Methylene blue; Spectrophotometry

- Research Ethics: The trial was conducted in accordance with the principles of the Declaration of Helsinki and approved by the Institutional Review Boards of all academic centers involved. All assessment subjects have signed an informed consent with protocol, including publication consent. - Conflict of Interest: Jan Krhut is a consultant Astellas Pharma. Except for that, no potential conflict of interest relevant to this article was reported.

\section{INTRODUCTION}

The use of onabotulinumtoxinA (onaBTA) has become a standard method of treatment in patients with neurogenic detrusor overactivity (NDO) refractory to anticholinergic therapy or for those that experience severe adverse effects. The number of patients treated using onaBTA for idiopathic detrusor overactivity (IDO) increased in recent years as well. Several clinical studies

Corresponding author: Vladimír Šámal (iD https://orcid.org/0000-0002-0081-9986 Department of Urology, Regional Hospital Liberec, Husova 10, 46063 Liberec, Czech Republic

E-mail: vladimir.samal@nemlib.cz

Submitted: August 27, 2019 / Accepted after revision: January 1, 2020 
documented high efficacy of endoscopic injection of onaBTA in increasing cystometric capacity, reducing detrusor pressure, and improving continence and quality of life $[1,2]$. Due to the nature of the drug administration, it is reasonable to assume that certain loss of the onaBTA solution occurs during the procedure. This could be caused by effusion into the perivesical fat or into the bladder lumen. Extravesical loss of onaBTA was investigated by Mehnert et al. [3], who demonstrated that up to $18 \%$ of injected onaBTA could be detected in perivesical tissue immediately after the procedure. Intravesical loss of onaBTA has not yet been investigated.

In clinical practice, a direct method for measuring onaBTA loss has not yet been developed. Analytical methods commonly used do not yield accurate results when measuring small volumes. In these cases, it is necessary to utilize an indirect measurement method that involves a substance that can be detected in order to yield reliable results.

The aim of this study was to determine the intravesical loss of onaBTA using spectrophotometry of methylene blue (MB) added to the reconstituted drug and subsequently quantified in the total volume of irrigation fluid used during the procedure. $\mathrm{MB}$ is an organic substance that is used as a dye in many medical fields, mostly as a diagnostic agent. An indisputable advantage to using $\mathrm{MB}$ is its ability to stain at very low concentrations, producing quantifiable measurements.

\section{MATERIALS AND METHODS}

The study was performed between July 2017 and June 2018 in 2 lower urinary tract dysfunction tertiary referral centers. The trial was conducted in accordance with the principles of the Declaration of Helsinki and approved by the Institutional Review Boards of all academic centers involved.

\section{Patients}

The study group was consisted of 48 patients (38 men, 10 women) with NDO. The mean age of the group was $36.6 \pm 8.9$ years. Forty-two patients had spinal injury-induced NDO and 6 patients suffered from NDO secondary to multiple sclerosis. Eligible patients who met all inclusion criteria were confirmed to be over the age of 18 , and were found to have experienced a failed pharmacological treatment approach due to low efficacy or significant adverse effects. Exclusion criteria included pelvic tumors, previous radical surgery of the small pelvis, cystolithiasis, indwelling catheter, low detrusor compliance, and clinically significant urinary tract infection.

\section{Procedure}

The dose of 200 units of onaBTA (Allergan Inc., Westport, Ireland) was reconstituted in $12 \mathrm{~mL}$ of saline and divided into 3 syringes of $4 \mathrm{~mL}$ each. To each syringe, $6 \mathrm{~mL}$ of stock solution of $\mathrm{MB}$, consisting of $2 \mathrm{~mL}$ of $2.5 \% \mathrm{MB}$ diluted in $100 \mathrm{~mL}$ of $0.9 \%$ saline solution, was added. OnaBTA was applied using the 30 -injection site template (6.7 U/mL per injection) excluding the trigone. The procedures were performed under general or spinal anesthesia using a rigid, $21 \mathrm{~F}$ cystoscope and flexible 23-G endoscopic needle. In an effort to reach the detrusor muscle, a needle was inserted perpendicular to the bladder wall, to a depth of 3-4 mm and injected $1 \mathrm{~mL}$ of onaBTA. Two experienced urologists performed all procedures using an identical injection protocol. All irrigation fluid (saline solution) used throughout the procedure was collected, the total volume was recorded and a 10-mL sample was sent for spectrophotometry analysis.

\section{Determination of the Amount of onaBTA in the Irrigation Fluid}

It is impossible to measure the concentration of onaBTA in the solution directly. The method used in this study was based on the assumption that the amount of onaBTA in the irrigation fluid after administration is proportionate to the concentration of $\mathrm{MB}$ in the same solution. Even very low amounts of $\mathrm{MB}$, when added to a clear solution, results in color change that can be quantified by spectrophotometry (UV-VIS Cintra 202, GBC Scientific Equipment Pty Ltd., Dandenong, Australia). The light passing through the MB solution is partially absorbed. Absorbance is the product of the extension coefficient $(\varepsilon)$, the length

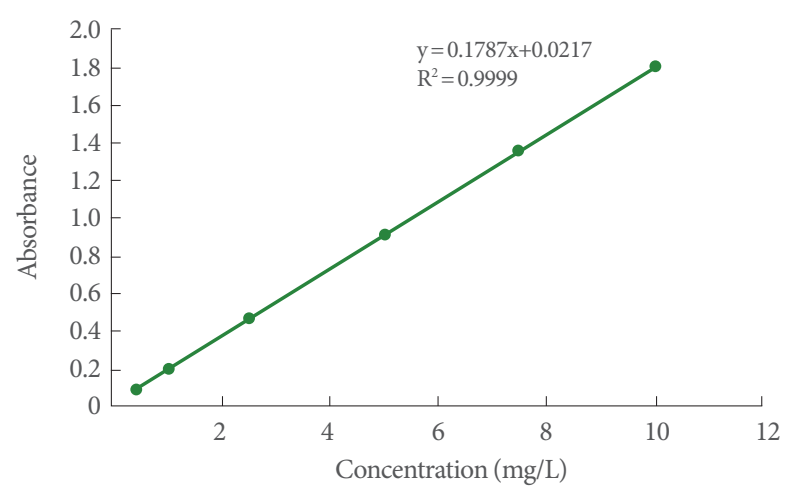

Fig. 1. Calibration graph of the absorbance of methylene blue. 
of the cuvette used for the measurement $(l)$, and the concentration of the solution $(c)$. To estimate the extension coefficient, a calibration graph was constructed using several absorbance measurements in a series of solutions with known MB concen-

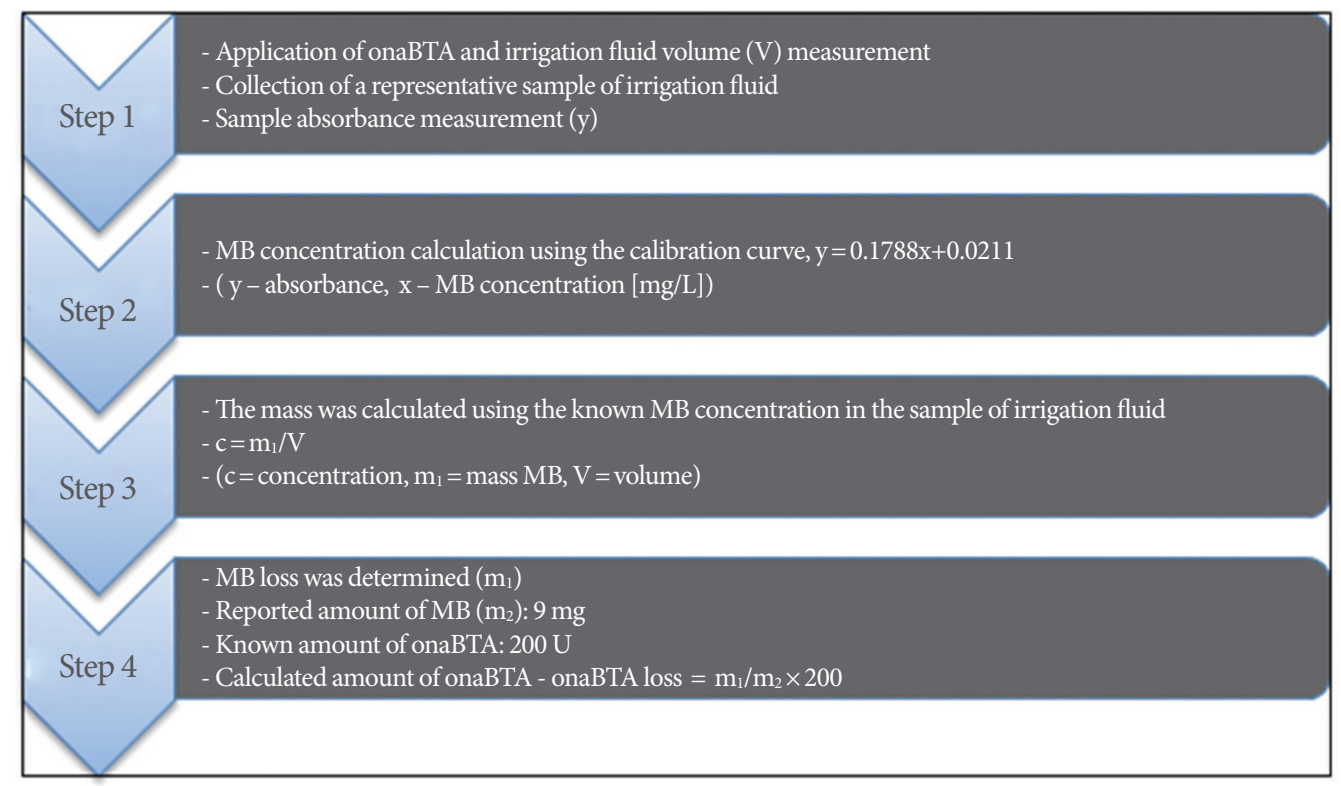

Fig. 2. Flowchart of calculation. onaBTA, onabolunumtoxinA; MB, methylene blue.
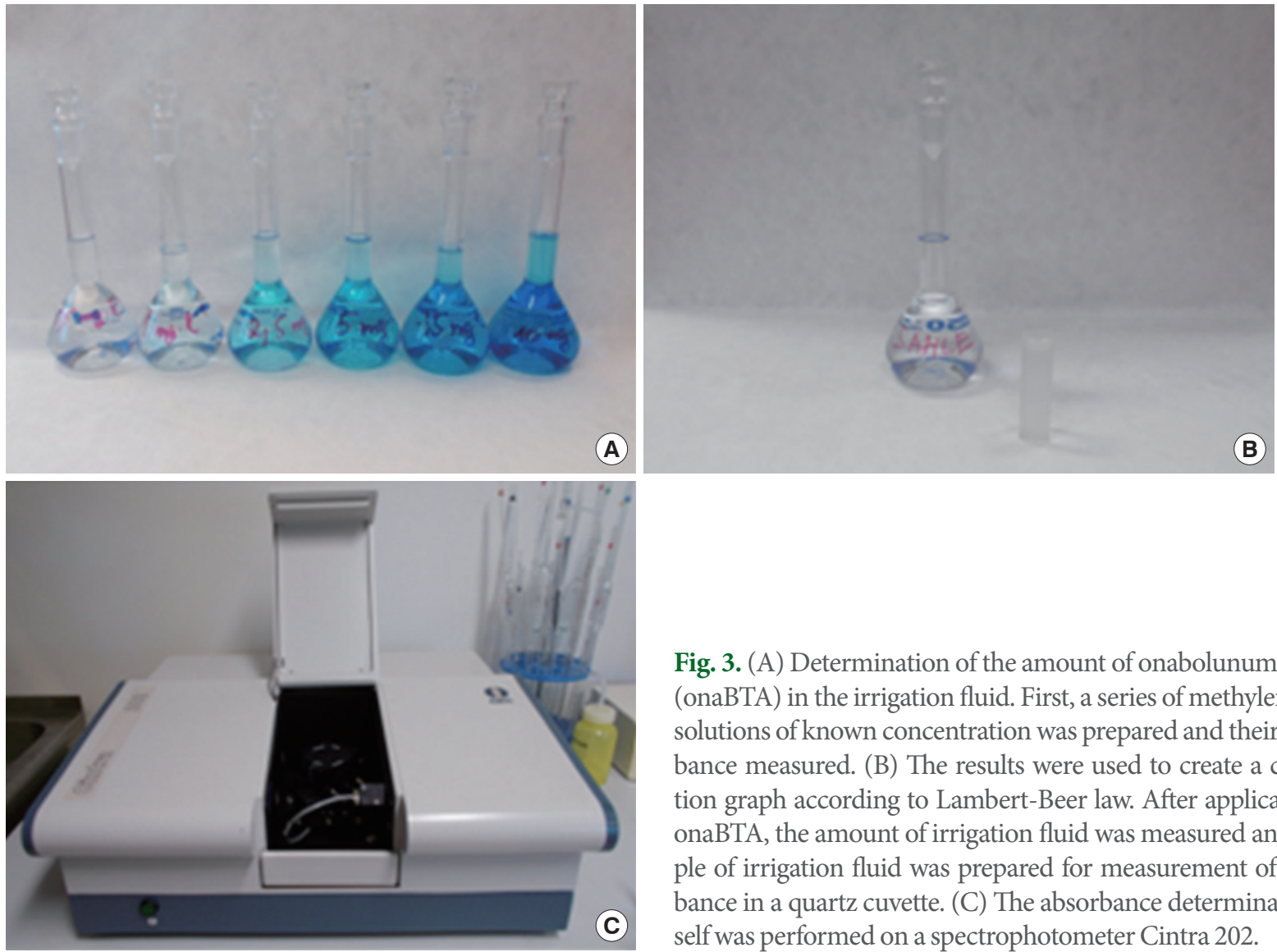

Fig. 3. (A) Determination of the amount of onabolunumtoxinA (onaBTA) in the irrigation fluid. First, a series of methylene blue solutions of known concentration was prepared and their absorbance measured. (B) The results were used to create a calibration graph according to Lambert-Beer law. After application of onaBTA, the amount of irrigation fluid was measured and sample of irrigation fluid was prepared for measurement of absorbance in a quartz cuvette. (C) The absorbance determination itself was performed on a spectrophotometer Cintra 202. 
trations. The correlation of absorbance and concentration for $\mathrm{MB}$ in saline solution is almost linear $\left(\mathrm{R}^{2}=0.9999\right)$. In our case, the equation of the calibration graph was $y=0.1788 x+0.0211$, where $y$ is the absorbance and $x$ is the MB concentration (Fig. 1). When measuring the absorbance of a solution of unknown concentration, the MB concentration can be subtracted from the graph using the curve equation.

After calculating the $\mathrm{MB}$ concentration in the irrigation fluid, the MB loss can be determined using the following concentration equation, $c=m / V$, where $c$ is the MB concentration, $m$ is the weight of $\mathrm{MB}$ and $V$ is the irrigation fluid volume.

Fig. 2 reports the loss of onaBTA, calculated using the known amount of $\mathrm{MB}$ administered and the amount of administered onaBTA. The procedure for measuring the onaBTA loss is summarized in Fig. 3.

\section{Statistical Analysis}

The results are reported as mean \pm standard deviation. Calculated values of loss of onaBTA exceeding an interquartile span of more than 1.5 times were considered outliers.

\section{RESULTS}

A total of 45 patients were included in the final analysis. During the study, we did not experience any complications or side effects. The mean volume of irrigation fluid was $603.33 \pm 400.14$ $\mathrm{mL}$. A mean absorbance value of the irrigation fluid samples was $0.14 \pm 0.12$ representing a $\mathrm{MB}$ concentration (MB intraluminal loss) of $0.19 \pm 0.18 \mathrm{mg} / \mathrm{L}$. The mean loss of onaBTA per application was calculated to be $4.14 \pm 4.11$ units, ranging from 0 to 14.5 units (Table 1, Fig. 4). We detected 3 outliers. These were reported in Table 1, but excluded from the final analysis. The extent of onaBTA loss did not significantly impact the efficacy of treatment in the 3 outliers. All outliers required repeat therapy and the duration of their response did not correlate with onaBTA loss. Thus, we did not consider the degree of onaBTA loss to be a predictor of the overall therapeutic effects.

\section{DISCUSSION}

Intradetrusor injection of onaBTA represents a standard method of onaBTA administration [4-6]. An increasing number of NDO patients is receiving onaBTA treatment, however, all aspects of this procedure have not yet been standardized and treatment outcomes remain variable. Among other factors, this variability could be attributed to onaBTA loss during injection.

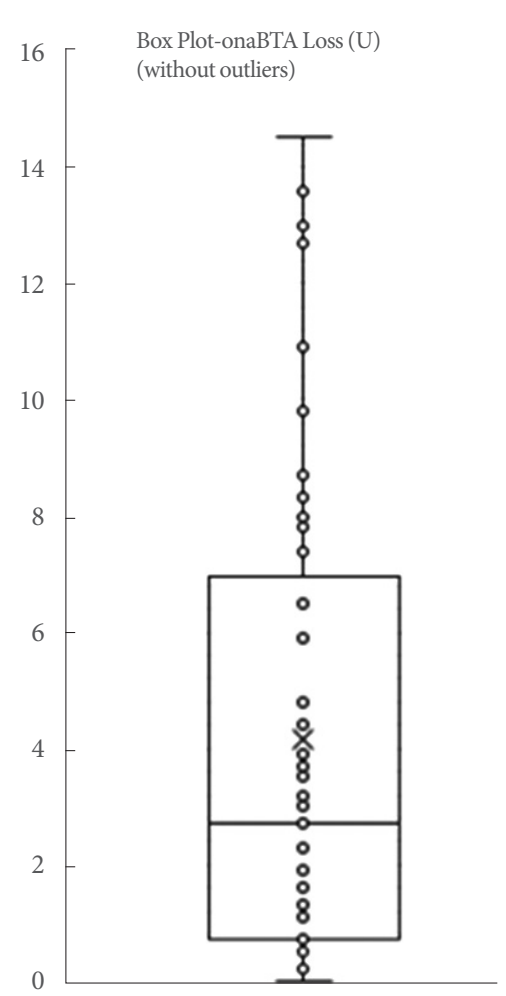

Fig. 4. Box plot illustrating onabotulinumtoxinA (onaBTA) loss (outliers excluded).

Table 1. Intravesical loss of onaBTA $(\mathrm{n}=45)$

\begin{tabular}{|c|c|c|c|c|c|c|c|c|}
\hline Variable & Mean & SEM & Median & Range & IQR & \multicolumn{3}{|c|}{3 Outliers } \\
\hline OnaBTA loss (U) & $4.14 \pm 4.11$ & 0.61 & 2.7 & $0-14.5$ & $0.7-6.95$ & 92.8 & 22.7 & 24.8 \\
\hline Washout volume (mL) & $603.33 \pm 400.14$ & 59.65 & 535 & $165-2,800$ & $400-749$ & 1,290 & 610 & 750 \\
\hline Washout concentration (mg/L) & $0.39 \pm 0.52$ & 0.08 & 0.24 & $0-2.96$ & $0.06-0.52$ & 3.24 & 1.68 & 1.49 \\
\hline Absorbance & $0.14 \pm 0.12$ & 0.02 & 0.11 & $0.02-0.56$ & $0.05-0.18$ & 0.6 & 0.32 & 0.29 \\
\hline Methylene blue loss (mg) & $0.19 \pm 0.18$ & 0.03 & 0.12 & $0-0.65$ & $0.03-0.31$ & 4.18 & 1.02 & 1.12 \\
\hline
\end{tabular}

OnaBTA, onabotulinumtoxinA; SEM, standard error of the mean; IQR, interquartile. 
Only one study has addressed this issue thus far. Mehnert et al. [3], evaluated the extravesical loss of onaBTA in 6 patients. They used the standard intradetrusor injection of onaBTA labeled with the contrast agent, gadopentetate. Immediately after administration, magnetic resonance imaging was performed. They were able to detect $82.4 \%$ of the administered agent in the detrusor, with the remaining volume detectable in perivesical fat. Interestingly, even with a significant amount of onaBTA detected outside the target distribution area, no side effects were reported.

The possibility of the intraluminal onaBTA loss has not been studied yet. Therefore, we designed a trial to address this issue. In this study, we assessed the most widely used technique of intradetrusor injection and detected minimal intraluminal loss ( $<3 \%$ of total dose). We recognize that there are several factors that could affect this. Among others, these include the number of injections, volume/concentration of a single injection, needle size, detrusor thickness and composition, and submucosal versus intradetrusor injection.

Most authors described studies using a 30-detrusor injection scheme. Others described treatment using 10-40 injections [7]. It is reasonable to assume that more injection points will result in a larger loss of the active compound. One study, however, addressed this hypothesis by comparing clinical outcomes using 10- and 30-injection sites. It reported no significant difference [1].

The reported total volume of the solution used for onaBTA reconstitution ranges from $3 \mathrm{~mL}$ to $30 \mathrm{~mL}$. A concentration of $6.7 \mathrm{U} / \mathrm{mL}$ per injection is the most commonly used $[5,6]$. Varying concentrations per single injection have been reported as well ranging from $10 \mathrm{U} / 0.5 \mathrm{~mL}$ to $10 \mathrm{U} / 0.1 \mathrm{~mL}$ [8]. A comparative study has not been conducted to date.

The standard 23-G endoscopic needle used in this study has been used most widely. It can be assumed that the use of a smaller needle might reduce the loss of the drug, however, with a small needle diameter, the mechanical stability of the onaBTA could be impaired [9].

The variable thickness and composition of the bladder wall (e.g., increased collagen content), which depends on sex, age, bladder filling, and functional condition of the bladder, represent additional factors affecting the accuracy of onaBTA delivery [10]. There are 2 possible mechanisms that could cause onaBTA loss into the bladder lumen; backflow along the needle tract during injection and leak through the needle deployment site immediately after injection. Based on our observation of an increase of visible MB leak in cases where the needle was removed soon after completion of injection, we recommend leaving the needle in the tissue for 3-5 seconds after injection to facilitate diffusion of the injected agents. Similarly, injection into the trabeculation of a neurogenic bladder results in larger backflow compared to injection into the adjacent tissue. This is most likely due to increased collagen content. It was previously documented, that the type of tissue in which the opening of the needle is placed has a large effect on the ability of diffusion. Differing resistances of tissue types have been shown to cause variable degrees of backflow of the injected substance along the needle tract [11].

Bladder filling at the time of injection may have a similar effect on backflow. According to our findings, the optimal intravesical volume at the time of injection is $300-400 \mathrm{~mL}$. Larger volumes are associated with increased intravesical pressure and bladder wall distension, which can ultimately affect diffusion of the drug and increase risk of backflow. To more accurately measure onaBTA loss, we measured both volume of irrigation fluid from the cystoscopy suction device as well as any volume that leaked from the cystoscope along the application needle, which was collected in a plastic bag placed under the cystoscope.

Suburothelial administration of onaBTA is an alternative to administration of onaBTA into the detrusor. Suburothelial administration can be visually well-controlled endoscopically. The experience has shown the same efficacy as injection into the detrusor $[12,13]$, however, no data reporting the loss of onaBTA during suburothelial administration are available.

Despite these variables, the aspect of intraluminal onaBTA loss during injection has not been previously evaluated. Our study is the first to deal with intravesical loss of onaBTA during administration. We used a unique absorbance assay for intraluminal onaBTA leak quantification, which proved practical and reliable and could be used in different studies addressing efficacy of tissue injection. Strengths of this study include use of this original laboratory technique, multicenter study design and the fact that it addresses a significant issue that has not yet been studied. The fact that, in $98.5 \%$ of study subjects, the loss of onaBTA into the bladder lumen did not exceed 3\% supports conclusiveness of our data. The occurrence of 3 outliers is difficult to explain. This was likely due to the increased intensity of hematuria, which is known to have an impact on measured absorbance values (increase absorbance), following the application of onaBTA. The design of the study did not primarily eval- 
uate the effectiveness of the treatment, it was focused on assessing the loss in the application of onaBTA. However, the duration of treatment response did not differ significantly between the cohort and outliers.

Also noteworthy are the analytical techniques used to determine onaBTA loss. The indirect method of monitoring drug loss has its limitations, including the selected dye to be controlled. There is a relatively large difference in weight of an $\mathrm{MB}$ molecule and onaBTA. The size of the molecule generally affects the rate of penetration through a membrane. For this study, penetration of $\mathrm{MB}$ and onaBTA is expected to be comparable. Determining the rate of penetration between onaBTA and $\mathrm{MB}$ is very problematic, likely yielding inaccurate data. The concentration of onaBTA and MB in the lavage at the start of the study would have to be measured to determine the conversion factor. Since the amount of onaBTA injected is very small, it is beyond the scope of its quantitative determination.

Determining the extinction coefficient by measuring the absorbance of a series of solutions at known concentrations is a common analytical practice. Only the linear portion of the curve is used for clinical practice.

Possible drug interactions between onaBTA and MB have not been studied in the clinical setting. Szczypior et al. [14] used MB to assess the distribution of onaBTA in the treatment of IDO patients. In the in vitro phase of this study, a pharmacological interaction between onaBTA and MB was not observed.

A limitation of this study is the lack of data correlating the lost onaBTA with clinical effects in individual patients. It would be valuable to show a reduced effect in patients with large onaBTA leak, however, in our study, this occurred very rarely (only 3 outliers). There are several future directions that we plan to pursue in the future. The most valuable of which will be a study comparing the loss of onaBTA in intradetrusor versus suburothelial administration and a comparison between NDO and IDO patients.

In conclusion, we have shown reproducible data supporting that intravesical loss of MB in an NDO patient during intradetrusor injection is less than $3 \%$ of the administered dose. This likely reflects the amount of onaBTA detected in the irrigation fluid.

\section{AUTHOR CONTRIBUTION}

- Conceptualization: VS

- Data curation: JMü

· Formal Analysis: JMü
- Investigation: $J K, V P, V S$

- Methodology: JMü

- Project Administration: VS, JM

-Writing - Original Draft: VS, JK

-Writing - Review \& Editing: VS, JK, JM, JMü

\section{REFERENCES}

1. Mangera A, Apostolidis A, Andersson KE, Dasgupta P, Giannantoni A, Roehrborn C, et al. An updated systematic review and statistical comparison of standardised mean outcomes for the use of botulinum toxin in the management of lower urinary tract disorders. Eur Urol 2014;65:981-90.

2. Kennelly M, Dmochowski R, Schulte-Baukloh H, Ethans K, Del Popolo G, Moore C, et al. Efficacy and safety of onabotulinumtoxi$\mathrm{nA}$ therapy are sustained over 4 years of treatment in patients with neurogenic detrusor overactivity: final results of a long-term extension study. Neurourol Urodyn 2017;36:368-75.

3. Mehnert U, Boy S, Schmid M, Reitz A, von Hessling A, Hodler J, et al. A morphological evaluation of botulinum neurotoxin A injections into the detrusor muscle using magnetic resonance imaging. World J Urol 2009;27:397-403.

4. Schurch B, Stöhrer M, Kramer G, Schmid DM, Gaul G, Hauri D. Botulinum-A toxin for treating detrusor hyperreflexia in spinal cord injured patients: a new alternative to anticholinergic drugs? Preliminary results. J Urol 2000;164(3 Pt 1):692-7.

5. Cruz F, Herschorn S, Aliotta P, Brin M, Thompson C, Lam W, et al. Efficacy and safety of onabotulinumtoxinA in patients with urinary incontinence due to neurogenic detrusor overactivity: a randomised, double-blind, placebo-controlled trial. Eur Urol 2011;60: 742-50.

6. Ginsberg D, Gousse A, Keppenne V, Sievert KD, Thompson C, Lam W, et al. Phase 3 efficacy and tolerability study of onabotulinumtoxinA for urinary incontinence from neurogenic detrusor overactivity. J Urol 2012;187:2131-9.

7. Del Popolo G, Filocamo MT, Li Marzi V, Macchiarella A, Cecconi F, Lombardi G, et al. Neurogenic detrusor overactivity treated with english botulinum toxin a: 8-year experience of one single centre. Eur Urol 2008;53:1013-19.

8. Rapp DE, Lucioni A, Katz EE, O'Connor RC, Gerber GS, Bales GT. Use of botulinum-A toxin for the treatment of refractory overactive bladder symptoms: an initial experience. Urology 2004;63:1071-5.

9. Dressler D, Bigalke H. Reconstituting botulinum toxin drugs: shaking, stirring or what? J Neural Transm (Vienna) 2016;123:523-5.

10. Silva JA, Gonsalves Mde C, de Melo RT, Carrerette FB, Damião R. 
Association between the bladder wall thickness and urodynamic findings in patients with spinal cord injury. World J Urol 2015;33: 131-5.

11. Plante MK, Gross AL, Folsom JB, Zvara P. Diffusion properties of transurethral intraprostatic injection. BJU Int 2004;94:1384-8.

12. Kuo HC. Therapeutic effects of suburothelial injection of botulinum a toxin for neurogenic detrusor overactivity due to chronic cerebrovascular accident and spinal cord lesions. Urology 2006;67:
232-6.

13. Krhut J, Samal V, Nemec D, Zvara P. Intradetrusor versus suburothelial onabotulinumtoxinA injections for neurogenic detrusor overactivity: a pilot study. Spinal Cord 2012;50:904-7.

14. Szczypior M, Połom W, Markuszewski M, Ciura K, BuszewskaForajta $\mathrm{M}$, Jacyna J, et al. Overactive bladder treatment: application of methylene blue to improve the injection technique of onabotulinum toxin A. Scand J Urol 2017;51:474-8. 